\title{
Is supratotal resection achievable in low-grade gliomas? Feasibility, putative factors, safety, and functional outcome
}

\author{
Marco Rossi, MD, ${ }^{1}$ Federico Ambrogi, PhD, ${ }^{2}$ Lorenzo Gay, MD, ${ }^{1}$ Marcello Gallucci, PhD, ${ }^{3}$ \\ Marco Conti Nibali, MD, ${ }^{1}$ Antonella Leonetti, PhD, ${ }^{1}$ Guglielmo Puglisi, PhD, ${ }^{1}$ \\ Tommaso Sciortino, MD, ${ }^{1}$ Henrietta Howells, PhD, ${ }^{1}$ Marco Riva, MD, ${ }^{4}$ Federico Pessina, MD, ${ }^{5}$ \\ Pierina Navarria, MD, ${ }^{6}$ Ciro Franzese, MD, ${ }^{6}$ Matteo Simonelli, MD, ${ }^{7,8}$ Roberta Rudà, MD, ${ }^{9}$ and \\ Lorenzo Bello, MD'
}

${ }^{1}$ Neurosurgical Oncology Unit, Department of Oncology and Hemato-Oncology, Università degli Studi di Milano, Humanitas Research Hospital, IRCCS; 'Laboratory of Medical Statistics, Biometry and Epidemiology "G.A. Maccararo," Department of Clinical Sciences and Community Health, Università degli Studi di Milano; ${ }^{3}$ Statistics, Department of Psychology, Università Milano-Bicocca; ${ }^{N}$ Neurosurgical Oncology Unit, Department of Medical Biotechnologies and Translational Medicine, Università degli Studi di Milano, Humanitas Research Hospital, IRCCS; 5 Neurosurgical Oncology Unit, Humanitas Research Hospital, IRCCS; ${ }^{6}$ Radiation Oncology, Humanitas Research Hospital, IRCCS; ${ }^{\circ}$ Oncology, Humanitas Research Hospital, IRCCS, Milan; ${ }^{8}$ Department of Biomedical Science, Humanitas University, Rozzano, Milan; and ${ }^{9}$ Neuro-Oncology Unit, Città della Salute e della Scienza, Università di Torino, Italy

OBJECTIVE Surgery for low-grade gliomas (LGGs) aims to achieve maximal tumor removal and maintenance of patients' functional integrity. Because extent of resection is one of the factors affecting the natural history of LGGs, surgery could be extended further than total resection toward a supratotal resection, beyond tumor borders detectable on FLAIR imaging. Supratotal resection is highly debated, mainly due to a lack of evidence of its feasibility and safety. The authors explored the intraoperative feasibility of supratotal resection and its short- and long-term impact on functional integrity in a large cohort of patients. The role of some putative factors in the achievement of supratotal resection was also studied.

METHODS Four hundred forty-nine patients with a presumptive radiological diagnosis of LGG consecutively admitted to the neurosurgical oncology service at the University of Milan over a 5-year period were enrolled. In all patients, a policy was adopted to perform surgery according to functional boundaries, aimed at achieving a supratotal resection whenever possible, without any patient or tumor a priori selection. Feasibility, general safety, and tumor or patient putative factors possibly affecting the achievement of a supratotal resection were analyzed. Postsurgical patient functional performance was evaluated in five cognitive domains (memory, language, praxis, executive functions, and fluid intelligence) using a detailed neuropsychological evaluation and quality of life (QOL) examination.

RESULTS Total resection was feasible in $40.8 \%$ of patients, and supratotal resection in $32.3 \%$. The achievement of a supratotal versus total resection was independent of age, sex, education, tumor volume, deep extension, location, handedness, appearance of tumor border, vicinity to eloquent sites, surgical mapping time, or surgical tools applied. Supratotal resection was associated with a long clinical history and histological grade II, suggesting that reshaping of brain networks occurred. Although a consistent amount of apparently MRI-normal brain was removed with this approach, the procedure was safe and did not carry additional risk to the patient, as demonstrated by detailed neuropsychological evaluation and QOL examination. This approach also improved seizure control.

CONCLUSIONS Supratotal resection is feasible and safe in routine clinical practice. These results show that a long clinical history may be the main factor associated with its achievement.

https://thejns.org/doi/abs/10.3171/2019.2.JNS183408

KEYWORDS low-grade glioma; supratotal; functional outcome; neuropsychology; resection; clinical impact; oncology

ABBREVIATIONS AED = antiepileptic drug; AIC = Akaike Information Criterion; $\mathrm{Cl}=$ confidence interval; $\mathrm{DWI}$ = diffusion-weighted imaging; $\mathrm{EOR}=$ extent of resection; GTR = gross-total resection; $L G G$ = low-grade glioma; $O R=$ odds ratio; $Q O L=$ quality of life. SUBMITTED December 10, 2018. ACCEPTED February 22, 2019.

INCLUDE WHEN CITING Published online May 17, 2019; DOI: 10.3171/2019.2.JNS183408. 
I N low-grade gliomas (LGGs), the use of a brain mapping technique increases the percentage of patients in whom a total and subtotal resection can be achieved and decreases the incidence of postoperative permanent deficits, maintaining patient integrity. ${ }^{2,8,10,12,18}$ According to this functional approach, resection is performed until functional boundaries are encountered. ${ }^{5}$ Functional boundaries can be found within, at, or outside tumor borders as visible on volumetric FLAIR images. ${ }^{7}$ The first situation, in which functional boundaries are found inside of the tumor mass, allows a partial, or subtotal, removal. In an intermediate situation, gross-total resection (GTR) can be achieved. When, instead, functional boundaries are distinctly outside the tumor area, part of the apparently intact brain parenchyma is removed along with the tumor itself, and thus a supratotal resection is obtained. ${ }^{25}$ The rationale for performing a supratotal resection is based on the finding that diffuse LGGs infiltrate the parenchyma far beyond conventional MRI abnormalities, suggesting that LGG recurrence after surgery may be due to undetected glioma cells growing beyond MRI-defined abnormalities. ${ }^{19}$ Moreover, the tissue located at the tumor periphery plays a crucial role in epileptogenesis due to tumor-induced changes in peritumoral cortex. ${ }^{9,16}$ Therefore, the peritumoral area is considered a fundamental surgical target, along with the MRI-defined tumoral bulk. Although limited, encouraging studies on oncological longterm outcome of supratotal resection suggested a significant impact on late tumor growth control and histological transformation. ${ }^{11}$ The main criticism to this approach concerns its wide-scale applicability, because it is considered feasible only in a small, anecdotal number of cases, with small-volume tumors located in "low-risk" locations. Additional concerns regard its safety and functional impact, considering that it requires the removal of brain parenchyma beyond the tumor border with no abnormalities on standard MR images. Finally, it is not clear how far resection can be safely extended around the tumor mass, or whether tumor location, extension, and volume may affect the amount of tissue that can be safely removed. Conclusively, many open questions remain unanswered for this promising approach.

In this study, all patients with a presumptive radiological diagnosis of LGG consecutively admitted to our service over a 5-year period were studied. In all patients we adopted the policy to perform surgery according to functional boundaries, aiming to achieve a supratotal resection whenever possible, without any patient or tumor a priori selection. We assessed feasibility and general safety; we analyzed the clinically, radiologically, and intraoperatively related factors possibly associated with achievement and degree of supratotal resection; and finally, we investigated functional outcome based on an analysis of neuropsychological and quality of life (QOL) profiles.

\section{Methods Patients}

All patients admitted to the neurosurgical oncology service at the University of Milan between May 2011 and April 2016, harboring a radiological diagnosis of presump- tive LGG-i.e., a measurable FLAIR mass lesion without contrast enhancement on postgadolinium T1-weighted images - and candidates for resective surgery, were included. All procedures were approved by our Ethical Committee.

\section{Imaging}

The preoperative MRI protocol, acquired on a Siemens MAGNETOM Verio 3-T MRI machine, included: 1) axial 3D FLAIR imaging; 2) postgadolinium 3D T1-weighted imaging; and 3) diffusion-weighted imaging (DWI) and apparent diffusion coefficient DWI. Functional MRI/DTI fiber tracking images were available in most patients. Patients underwent both immediate (within 48 hours) and 2-month postoperative MRI (volumetric FLAIR and postgadolinium T1-weighted) to estimate the extent of resection (EOR). Immediate postoperative diffusion-weighted MRI scans were performed to rule out ischemia.

\section{Surgical Procedures}

Resection was accomplished according to functional boundaries, with the aim of achieving a supratotal resection whenever possible, without any patient or tumor (size, location, or extension) a priori selection. The craniotomy exposed the tumor area and a limited amount of surrounding tissue. Surgery was performed under asleep-awake-asleep anesthesia, or asleep anesthesia (according to clinical needs). Cortical mapping allowed us to determine the cortical safe entry zone. Resection was performed with the aid of a brain mapping and monitoring technique, ${ }^{7}$ using subcortical mapping intraoperative neurophysiology and, in most cases, neuropsychology to locate functional boundaries (motor, language, haptic, visual, and cognitive), which ultimately defined the resection margins. Resection was initiated at the tumor periphery and continued until functional boundaries were reached, independently from where they were located with respect to tumor borders visible on MRI. Once subcortical tracts were identified and the tumor functionally disconnected, the mass was removed (during asleep anesthesia), with the aid of motor mapping and motor-evoked potential/somatosensory-evoked potential EEG monitoring. All procedures were standardized and performed by the same surgeon (L.B.) with the aid of the first author (M.R.).

\section{Extent of Resection}

EOR corresponded to the percentage of tumor volume resected with respect to the preoperative volume, i.e., preoperative tumor volume - postoperative residual volume)/ preoperative tumor volume, evaluated using volumetric FLAIR imaging. ${ }^{23}$ Volumes were computed onto volumetric FLAIR imaging with manual segmentation using iPlan Cranial software (BrainLAB) by three blinded investigators (M. Rossi, M. Riva, and M.C.N.). Any FLAIR hyperintense signal abnormalities were included in the lesion load and reported in cubic centimeters. Total resection was achieved when EOR was equal to $100 \%$. A supratotal resection was defined as the complete removal of any signal abnormalities, with the volume of the postoperative cavity being larger than the preoperative tumor volume ${ }^{25}$ 

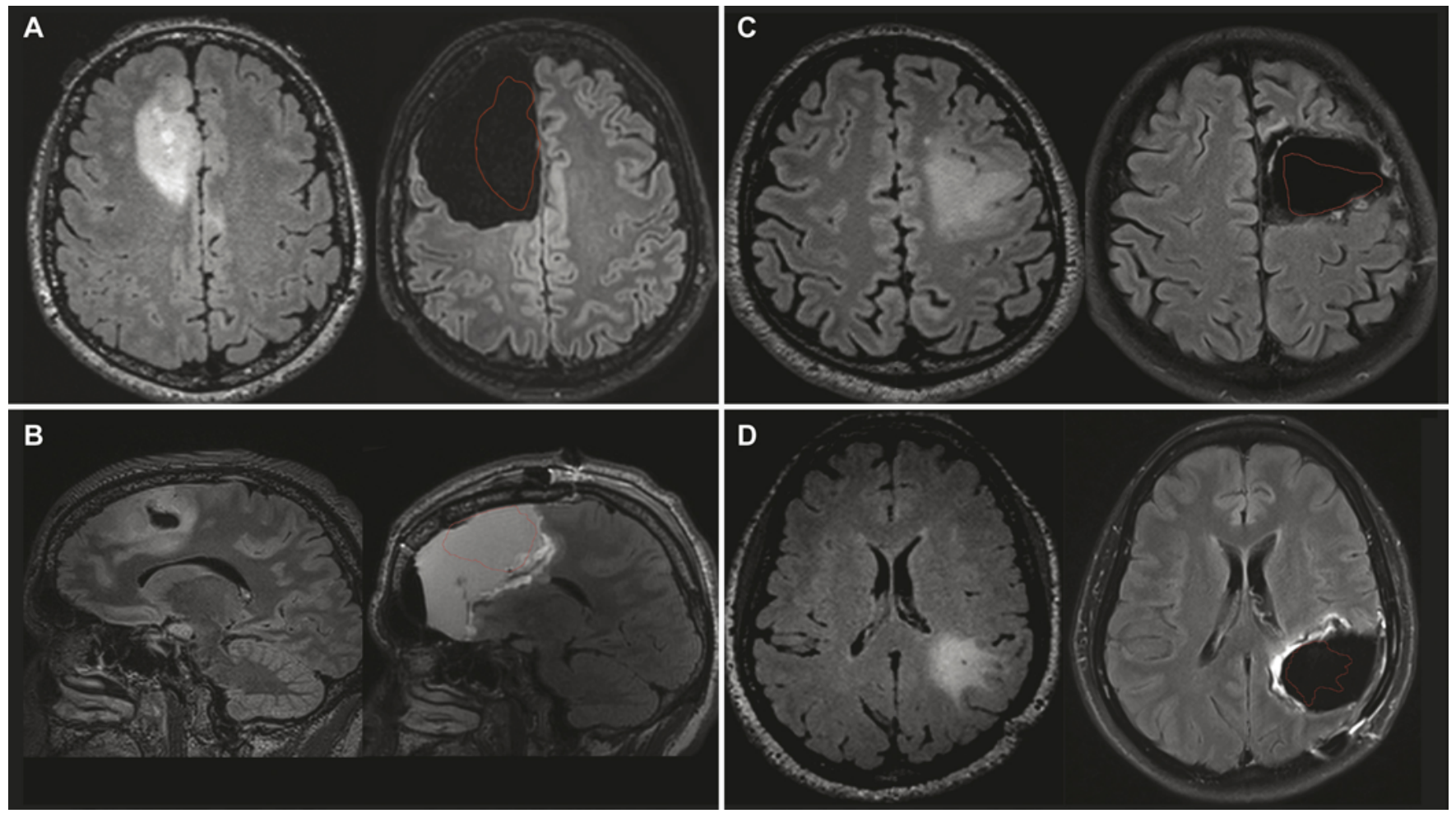

FIG. 1. Examples of supratotal resection. The tumor volume is highlighted in red using the semiautomated Smartbrush software (Elements, BrainLAB) and superimposed on the coregistered postoperative images. A: Example of supratotal resection in a case of nondominant frontal LGG (preoperative axial FLAIR image, left). Resection was accomplished according to motor, haptic, and cognitive boundaries, found far from the tumor border, enabling a supratotal tumor removal (2-month postoperative FLAIR image, right). The tumor volume was $20.7 \mathrm{~cm}^{3}$, resection cavity $80.8 \mathrm{~cm}^{3}$, and the calculated degree of supratotal resection was $390 \%$. B: Example of supratotal resection in a case of dominant frontal LGG involving the corpus callosum and characterized by irregular tumor borders (preoperative axial FLAIR image, left). Resection was performed according to motor, haptic, language, and cognitive boundaries, found outside the tumor borders. The anterior and middle portion of the corpus callosum was removed (2-month postoperative FLAIR image, right). The tumor volume was $20.7 \mathrm{~cm}^{3}$, resection cavity $128.5 \mathrm{~cm}^{3}$, and the calculated degree of supratotal resection was $620 \%$. C: Example of supratotal resection in a case of dominant frontal LGG involving the dorsal and ventral premotor areas (preoperative axial FLAIR image, left). Resection was performed according to motor, haptic, language, and cognitive boundaries, found outside the tumor border (2-month postoperative FLAIR image, right). Tumor volume was $17 \mathrm{~cm}^{3}$, resection cavity $42 \mathrm{~cm}^{3}$, and the calculated degree of supratotal resection was $240 \%$. D: Example of supratotal resection in a case of dominant parietal LGG with irregular tumor borders (preoperative axial FLAIR image, left). Resection was performed according to motor, language, haptic, and visual boundaries, found outside the tumor border (2-month postoperative FLAIR image, right). Tumor volume was $12.8 \mathrm{~cm}^{3}$, resection cavity $60.8 \mathrm{~cm}^{3}$, and the calculated degree of supratotal resection was $475 \%$. Figure is available in color online only.

(Fig. 1). To quantify supratotal resection, the volume of the surgical cavity was calculated using manual segmentation with iPlan Cranial software on MRI at 2 months after surgery, and this volume was compared with the preoperative tumor volume. ${ }^{25}$ Patients were stratified into 3 groups according to EOR (Fig. 2).

\section{Feasibility and General Safety}

Feasibility was assessed by calculating the number of cases in which a supratotal resection was achieved; measuring the duration of surgery (incision to closure); measuring mapping time (time to perform cortical/subcortical mapping); and occurrence during the awake phase of patient fatigue, or mapping abortion (due to patient discomfort or reduced collaboration). General safety was assessed by evaluating the number of immediate (new within the first week of surgery) and permanent (persistent at
1 month after surgery) neurological deficits, occurrence of intraoperative complications (e.g., intracavitary bleeding or ischemia), and operative mortality.

\section{Factors Affecting Supratotal Resection}

Among the factors possibly associated with the achievement of a supratotal resection, the following were investigated: 1) factors relating to the patient (age, sex, clinical history duration, incidental discovery, epilepsy history, handedness); 2) factors related to the tumor that were deducible by evaluating preoperative conventional MRI (location, volume, hemispheric dominance, involvement of functional sites at the cortical or subcortical level, tumor border [either well-defined or diffuse], and involvement of corpus callosum); 3) intraoperative factors such as location of subcortical functional borders (within, at the tumor border, or outside), or previous biopsy or recurrence; and 


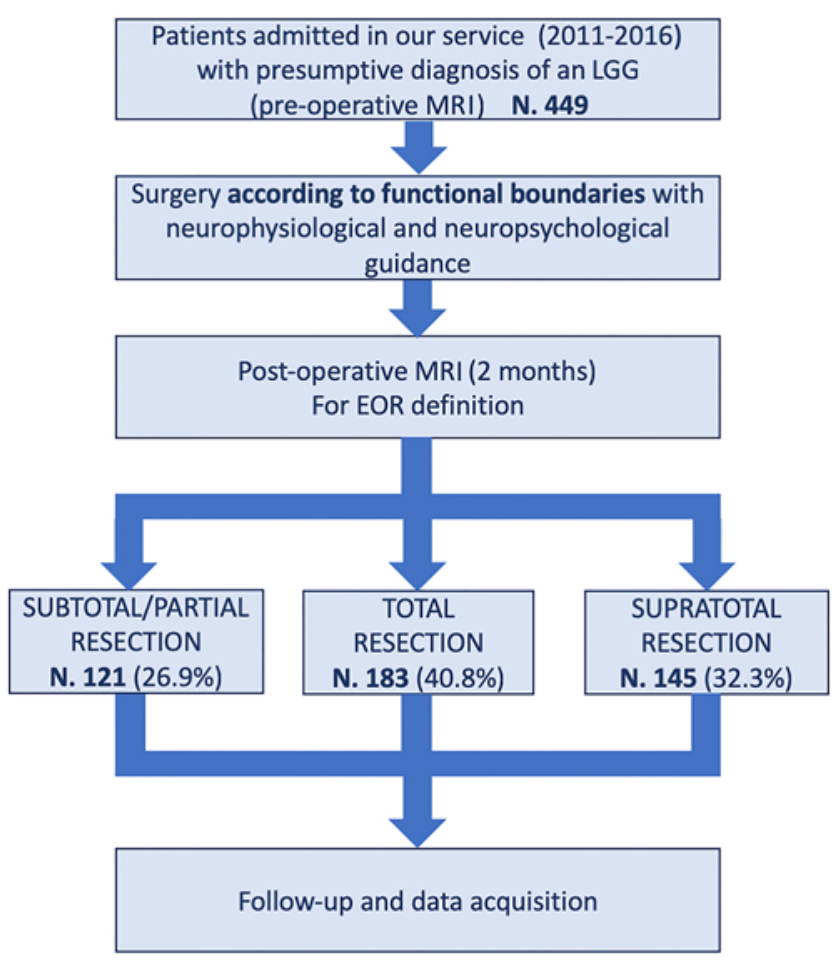

FIG. 2. Flowchart showing the process used for patient stratification. Patients were stratified into subtotal/partial, total, and supratotal resection groups. The number and respective percentage of patients included in each group are reported. Figure is available in color online only.

4) integrated molecular/histological diagnosis, histological grade, and $I D H I$ mutation.

Duration of clinical history was categorized as $<$ or $>$ 6 months. Epilepsy history was categorized as focal (and related to tumor functional sites, e.g., motor Jacksonian seizures in primary motor cortex tumors) or generalized (including focal followed by generalization). Handedness was assessed using the Edinburgh Handedness Inventory questionnaire. Tumor location was defined according to the lobe most involved (e.g., parietal, etc.). The frontal group included anterior (polar) and posterior (ventral premotor, dorsal premotor, supplementary motor, and pure motor) gliomas; paralimbic tumors included mostly insular gliomas. Tumor borders were evaluated on FLAIR, and referred to as well-defined or highly irregular., ${ }^{1,6}$ The distance between the tumor and brain sites anatomically related to main neurological functions (such as motor, language, visual, visuospatial, etc.) was computed on preoperative FLAIR imaging ${ }^{25}$ sites were considered infiltrated when at a distance $<5 \mathrm{~mm}$. The corpus callosum was considered infiltrated when the tumor reached or involved the corpus callosum on preoperative FLAIR imaging.

\section{Assessment of the Degree of Supratotal Resection}

The ratio between the surgical cavity and preoperative tumor volume defined the degree of supratotal resection, which was expressed as a percentage value..$^{25}$ The association between degree of supratotal resection and tumor volume was also investigated.
Cavity collapse was defined as any changes or deformation of the surgical cavity as a result of brain volume modifications that occurred as a consequence of resection, CSF drainage, and gravity. ${ }^{17,22}$ In deep (e.g., paralimbic) or posterior (e.g., parietal or posterior-temporal) locations, cavity collapse should be more evident as compared with frontal locations, due to the effect of gravity. For this reason, we assessed the effect of cavity collapse on the calculation of the degree of supratotal resection in different locations.

\section{Detailed Analysis of Functional Outcome}

The analysis of functional outcome was conducted on a subgroup of patients fulfilling the following criteria: 1) availability of both pre- and postoperative volumetric MRI; 2) EOR $\geq 100 \%$; 3) complete clinical history, comprehensive pre- and postoperative information about seizures and seizure control, antiepileptic drugs (AEDs), neurological deficits, and other treatments (previous surgery, adjuvant CT); 4) full molecular data; 5) neuropsychological evaluations performed preoperatively, and at 5 days and 1-3 months after surgery; and 6) availability of global performance status and ability and time to return to work. Neuropsychological data at 1 year and QOL assessment were also available in 60 of these patients.

\section{Neuropsychological Profile Evaluation}

The neuropsychological profile was evaluated with the Milano-Bicocca battery. ${ }^{20}$ Language, memory, praxis abilities, attentive and executive functions, and fluid intelligence were assessed. The score of each test was corrected for patient age and educational level to obtain a normalized value and converted to an equivalent score on a scale of five values from 0 to 4 ( 0 = pathologic, $1=$ inferior limit of normal performance, $2-4=$ different degrees of normal performance). The division of corrected scores into five different regions, each corresponding to an equivalent score, was based on the distribution of the scores observed in a control population; the first region (equivalent score = 0 ) corresponds to the worst 5 th percentile results observed in the control population, while the region on the opposite end (equivalent score $=4$ ) corresponds to the corrected scores in the control population between the median and the maximum score. The other three regions $(1,2$, and 3$)$ are defined between these two extreme regions, so that the ranges of the corrected scores observed are evenly spaced between the normality reference threshold and median. This conversion in equivalent scores allowed the comparison of test scores among different subjects.

\section{Global Performance and QOL Evaluation}

Global functional outcome was assessed by using measures of cognition, seizure activity and control, symptom severity and burden, neurological deterioration, global performance status, and ability and time to return to work. ${ }^{4}$ For detailed assessment of QOL, the QOL assessment and Hospital Anxiety and Depression Scale were available in 60 patients. $^{26}$

\section{Statistical Analysis}

To evaluate the association between feasibility of tu- 
mor resection with some putative risk factors, resection was categorized into 1 of 3 levels: partial/subtotal, total, and supratotal. A multinomial regression model (using total resection as the baseline) was considered. Results were expressed using two odds ratios (ORs) for each investigated variable: one considering the odds of supratotal versus total resection, and the other considering the odds of partial/subtotal versus total resection. The two ORs were collapsed into one if there was no evidence of difference according to the likelihood ratio test, indicating a variable of reduced clinical relevance. An initial screening of variables to be considered in the multivariable regression model was performed using univariable multinomial regression. Finally, a multivariable, partially unconstrained, multinomial logistic model was fitted. .,14 $^{2}$

Regression analysis was used to investigate the association between tumor volume and cavity volume. Generalized additive models for location, scale, and shape ${ }^{21}$ were used, allowing not only the mean but also the variance to depend on covariates, accounting for possible nonlinear effects using spline functions. The model allows one to estimate relevant percentiles of the cavity volume distribution as a function of tumor volume. The Akaike Information Criterion (AIC) was used to select spline complexity. ${ }^{24}$ The adjusted coefficient of determination $\left(\mathrm{R}^{2}\right)$ was used to evaluate the explained variation by the regression equations. Bootstrapping was also used to account for optimism in $\mathrm{R}^{2}$. The model selection phase (selection of the number of spline bases) was not included in the validation with bootstrapping.

To evaluate differences in degree of supratotal resection when comparing insular or parietal versus frontal locations, ANCOVA was used on tumor volume and location, regressing the difference between the cavity and tumor volume. To evaluate the association of resection with patient performance, quantitative variables were analyzed using ANOVA with resection groups (total vs supratotal) as independent variables. Qualitative variables were analyzed using logistic regression, with resection group as the independent variable.

Regarding neuropsychological test analysis, all test results were categorized as abnormal (equivalent score 0 or 1) or normal. A logistic regression was then used to evaluate the association with resection groups. Because neuropsychological tests were repeated in the follow-up evaluations over different time points, a random coefficients model was implemented. The random components of test response were modeled by allowing intercepts to vary across patients and across tests. ${ }^{4}$ Thus, the probability of a deficit in a test of each domain was modeled as a random-intercept generalized linear model, with patient and test as clustering variables, and time point of followup, resection group, and their interaction as fixed effects. The effects exerted on each domain by preoperative volume and histological-molecular profile were estimated on average, and in interaction with time of assessment.

\section{Results \\ Patients}

In the analyzed period, 449 patients with a presumptive radiological diagnosis of LGG and who were candidates for resective surgery were consecutively admitted. Patient clinical, radiological, and intraoperative features are listed in Table 1. Asleep-awake-asleep anesthesia was used in $94.5 \%$ of patients (Table 2) for language, cognitive, haptic, and visual mapping. Asleep anesthesia was used in cases of small, nondominant, anterior temporal pole tumor resection in which subcortical motor mapping defined the medial margin of resection. Integrated diagnosis revealed an oligodendroglioma in $51.4 \%$ of cases and an astrocytoma in $48.6 \%$. A low grade was diagnosed in $79.5 \%$ of cases. Of the high-grade tumors $(20.5 \%)$, the majority were grade III (18.7\%). The IDHI mutation was detected in $91.1 \%$, and wild-type in $8.9 \%$.

\section{Feasibility and General Safety of Supratotal Resection}

A total resection was obtained in 183 patients $(40.8 \%$, 95\% confidence interval [CI] 35.9\%-46.0\%), a supratotal resection in $145(32.3 \%, 95 \%$ CI $27.4 \%-37.5 \%)$, and a subtotal/partial resection in the remaining patients $(n=121)$. No significant differences in global surgical time and/or mapping (cortical/subcortical) time emerged between the resection groups. During the awake surgeries (the majority of procedures), the amount of patient fatigue was low and similar in all groups $(<1 \%)$ and no mapping abortion was observed (Table 2).

To assess safety, in all resection groups we analyzed the incidence of immediate or permanent neurological deficits and surgically related complications (Table 2). Acute postsurgical functional outcome assessment revealed new deficits (e.g., reduction of speech, slowness of movement), which recovered within 1-2 weeks. The incidence of permanent neurological deficits was low in all resection groups, but was significantly higher in patients in the subtotal/partial resection group in comparison to the total or supratotal groups $(6.6 \%$ vs $0.55 \%$ and $0.68 \%$, respectively, $\mathrm{p}<0.001$; Table 2). No postoperative mortality was observed.

\section{Achievement of Supratotal Resection}

The effect of various parameters was assessed (Table 3). Among patient-related factors, left-handedness and incidental discovery occurred in a few patients, and were therefore excluded from the analysis.

Considering univariate regressions, the association of sex, affected hemisphere, proximity to eloquent sites, and tumor volume with any resection group was not significant. Such variables were not included in the multivariable regression. Among clinical variables, a duration of clinical history longer than 6 months was strongly associated with the achievement of supratotal resection, i.e., a patient with a clinical history $>6$ months was approximately 24 times more likely to have a supratotal versus a total resection than a patient with a clinical history $<6$ months. Regarding seizures before surgery, this parameter was less clinically relevant, as the OR for generalized versus focal seizures was very large with respect to the achievement of partial/subtotal versus total and of supratotal versus total resection. Among imaging parameters, the involvement of the corpus callosum was associated with greater odds of 
TABLE 1. Clinical and imaging features

\begin{tabular}{|c|c|c|c|c|}
\hline \multirow[b]{2}{*}{ Category } & \multicolumn{3}{|c|}{ EOR } & \multirow[b]{2}{*}{$p$ Valuef } \\
\hline & Partial/Subtotal & Total & Supratotal & \\
\hline No. of patients, $n(\%)$ & $121(26.9)$ & $183(40.8)$ & $145(32.3)$ & \\
\hline Age, yrs & & & & 0.108 \\
\hline Median & 38.2 & 37.2 & 36.5 & \\
\hline Mean & 39.9 & 38.1 & 37.9 & \\
\hline Sex & & & & 0.091 \\
\hline Males & 73 & 119 & 77 & \\
\hline Females & 48 & 64 & 68 & \\
\hline Admission & & & & $<0.001$ \\
\hline Symptomatic & 121 & 178 & 131 & \\
\hline Incidental & 0 & 5 & 14 & \\
\hline Seizure, n (\%) & & & & $<0.001$ \\
\hline Generalized & $120(99.2)$ & $81(44.3)$ & $131(90.3)$ & \\
\hline Focal & $1(0.8)$ & $97(53.0)$ & $0(0)$ & \\
\hline Clinical history, n (\%) & & & & $<0.001$ \\
\hline$<6$ mos & $62(51.2)$ & $84(45.9)$ & $7(4.8)$ & \\
\hline$>6 \mathrm{mos}$ & $59(48.8)$ & $99(54.1)$ & $138(95.2)$ & \\
\hline Handedness & & & & 0.330 \\
\hline $\mathrm{Lt}$ & 3 & 1 & 1 & \\
\hline $\mathrm{Rt}$ & 118 & 182 & 144 & \\
\hline Dominant, n (\%) & & & & 0.041 \\
\hline Yes & $78(64.5)$ & $103(56.3)$ & $71(49)$ & \\
\hline No & $43(35.5)$ & $80(43.7)$ & $74(51)$ & \\
\hline Side, $n(\%)$ & & & & 0.019 \\
\hline $\mathrm{Lt}$ & $84(69.4)$ & $111(60.7)$ & $76(52.4)$ & \\
\hline $\mathrm{Rt}$ & $37(30.6)$ & $72(39.3)$ & $69(47.6)$ & \\
\hline Location, n (\%) & & & & $<0.001$ \\
\hline Frontal & $38(31.4)$ & $85(46.4)$ & $86(59.3)$ & \\
\hline Temporal & $18(14.9)$ & $33(18)$ & $28(19.3)$ & \\
\hline Parietal & $23(19)$ & $31(16.9)$ & $7(4.8)$ & \\
\hline Insular & $42(34.7)$ & $34(18.6)$ & $24(16.6)$ & \\
\hline Eloquent, $\mathrm{n}(\%)$ & & & & 0.029 \\
\hline Yes & $115(95)$ & $172(94)$ & $126(86.9)$ & \\
\hline No & $6(5)$ & $11(6)$ & $19(13.1)$ & \\
\hline MRI borders, $n(\%)$ & & & & 0.013 \\
\hline Compact & $23(19)$ & $62(33.9)$ & $36(24.8)$ & \\
\hline Diffuse & $98(81)$ & $121(66.1)$ & 109 (75.2) & \\
\hline Corpus callosum, n (\%) & & & & $<0.001$ \\
\hline Yes & $10(8.3)$ & $64(35)$ & $52(35.9)$ & \\
\hline No & $111(91.7)$ & $119(65)$ & $93(64.1)$ & \\
\hline Preop volume in $\mathrm{cm}^{3}$ & & & & 0.680 \\
\hline Min & 0.9 & 0.7 & 0.3 & \\
\hline Max & 385.5 & 366.6 & 278.0 & \\
\hline Median & 43.15 & 46.1 & 49.1 & \\
\hline Mean & 71.1 & 63.7 & 67.4 & \\
\hline \multicolumn{5}{|l|}{ Intraop factors } \\
\hline Functional borders, $\mathrm{n}(\%)^{*}$ & & & & $<0.001$ \\
\hline Inside FLAIR margin & $120(99.2)$ & $1(0.5)$ & $0(0)$ & \\
\hline At FLAIR margin & $1(0.8)$ & $180(98.4)$ & $0(0)$ & \\
\hline Outside FLAIR margin & $0(0)$ & $2(1.1)$ & $145(100)$ & \\
\hline Second surgery & & & & $<0.001$ \\
\hline No & 103 & 140 & 133 & \\
\hline Previous biopsy & 15 & 37 & 11 & \\
\hline Previous surgery & 3 & 6 & 1 & \\
\hline
\end{tabular}


» CONTINUED FROM PAGE 1697

TABLE 1. Clinical and imaging features

\begin{tabular}{|c|c|c|c|c|}
\hline \multirow[b]{2}{*}{ Category } & \multicolumn{3}{|c|}{ EOR } & \multirow[b]{2}{*}{$p$ Valuef } \\
\hline & Partial/Subtotal & Total & Supratotal & \\
\hline \multicolumn{5}{|l|}{ Intraop factors (continued) } \\
\hline Histology, n (\%)† & & & & 0.006 \\
\hline Oligodendroglioma & $62(51.2)$ & $80(43.7)$ & $89(61.4)$ & \\
\hline Astrocytoma & $59(48.8)$ & $103(56.3)$ & $56(38.6)$ & \\
\hline Histological grade, $\mathrm{n}(\%)$ & & & & $<0.001$ \\
\hline Low & $90(74.4)$ & $135(73.8)$ & $132(91)$ & \\
\hline High & $31(25.6)$ & 48 (26.2) & $13(9)$ & \\
\hline IDH1, n (\%) & & & & 0.021 \\
\hline Mutated & $117(96.7)$ & $161(88)$ & $131(90.3)$ & \\
\hline Wild-type & $4(3.3)$ & $22(12)$ & $14(9.7)$ & \\
\hline
\end{tabular}

${ }^{*}$ Functional borders indicate where the functional boundaries were detected at intraoperative subcortical functional mapping in relation to tumor FLAIR abnormalities.

† An oligodendroglioma was defined when an IDH1 mutation and 1p/19q codeletion were present. An astrocytoma was defined when a $1 p / 19 q$ codeletion was absent and an ATRX mutation was present. IDH1 status was determined by immunohistochemistry, in case of negative expression by mutational analysis. Codeletion was determined by fluorescent in situ hybridization; ATRX loss, p53 mutation, and Ki67 were determined by immunohistochemistry. For MGMT methylation status, DNA was extracted and determined by pyrosequencing.

$\ddagger$ The Fisher exact test and Kruskal-Wallis test were used to evaluate the hypothesis of no association with the resection group.

partial/subtotal versus total resection, while there was no evidence of association for the odds of supratotal versus total resection. The association with tumor border was not as clinically relevant. Regarding tumor location, the likelihood of reaching a total or supratotal resection was higher in frontal and temporal tumors, but without a significant difference between total or supratotal resection groups. The odds of insular location having a supratotal resection were smaller than the odds of frontal locations, but without a significant difference between total or supratotal groups. Parietal location was associated with smaller odds (0.26) of having a supratotal resection. Among histological/molecular parameters, IDHI mutation was not significant, whereas diagnosis of LGG and oligodendroglioma correlated with the achievement of supratotal resection. As for surgical parameters, supratotal resection appeared per- fectly associated with the identification of all functional sites outside the tumor border, whereas previous biopsy or recurrence was not as clinically relevant.

\section{Degree of Supratotal Resection}

The degree of supratotal resection was calculated by comparing tumor volume on preoperative FLAIR imaging and surgical cavity volume on postoperative FLAIR imaging. The degree of supratotal resection varied between $105.6 \%$ and $4371.5 \%$ (median 320.2\%). Regarding the association between tumor volume and cavity volume, a regression model with 3 spline bases was selected using AIC. The adjusted $\mathrm{R}^{2}$ was 0.63 corrected for optimism. The association was significant (Fig. 3).

To assess the effect of cavity collapse on the calculation of degree of supratotal resection, we compared mea-

TABLE 2. Clinical and surgical features

\begin{tabular}{lccccc}
\hline & General Population & Partial/Subtotal & Total & Supratotal & $p$ Value \\
\hline No. of patients, $\mathrm{n}(\%)$ & $449(100)$ & $121(27)$ & $183(40.8)$ & $145(32.3)$ & \\
\hline Asleep-awake-asleep, $\mathrm{n}(\%)$ & $424(94.4)$ & $112(92.6)$ & $173(94.5)$ & $139(95.9)$ & 0.503 \\
\hline Mean duration of surgery \pm SD, min & $229 \pm 18$ & $225 \pm 19.4$ & $234 \pm 21$ & $228 \pm 19$ & 0.532 \\
\hline Mean mapping time \pm SD, min & $40 \pm 18$ & $39.5 \pm 17.3$ & $39.5 \pm 15$ & $41.5 \pm 12$ & 0.492 \\
\hline Intraop complications, $\mathrm{n}(\%)^{*}$ & $4(0.9)$ & $2(1.6)$ & $1(0.5)$ & $1(0.7)$ & 0.575 \\
\hline Mappings aborted & 0 & 0 & 0 & 0 & $\mathrm{NA}$ \\
\hline Patient fatigue, $\mathrm{n}(\%)$ & 8 & $4(3.3)$ & $3(1.6)$ & $1(0.7)$ & 0.270 \\
\hline Operative deaths & 0 & 0 & 0 & 0 & $\mathrm{NA}$ \\
\hline Immediate deficits, $\mathrm{n}(\%)$ & $399(88.9)$ & $111(91.7)$ & $161(88)$ & $127(87.6)$ & 0.498 \\
\hline Permanent deficits, $\mathrm{n}(\%)$ & $9(2)$ & $8(6.6)$ & $1(0.55)$ & $1(0.69)$ & $<0.001$ \\
\hline
\end{tabular}

NA = not applicable.

* Intraoperative complications included intracavitary bleeding $(n=2)$, ischemia $(n=1)$, and infection $(n=1)$. 
TABLE 3. Multinomial logistic model

\begin{tabular}{|c|c|c|c|c|c|c|}
\hline \multirow[b]{2}{*}{ Variable } & \multicolumn{4}{|c|}{ Univariable Regression } & \multicolumn{2}{|c|}{ Multivariable Regression } \\
\hline & OR $(95 \% \mathrm{Cl})$ & $\begin{array}{l}\text { OR }(95 \% \mathrm{Cl}) \\
\text { Proportional }\end{array}$ & $\begin{array}{l}\text { Proportional } \\
\text { Odds ( } p \text { Value) }\end{array}$ & $p$ Value & OR $(95 \% \mathrm{Cl})$ & $\mathrm{p}$ Value \\
\hline \multirow[t]{2}{*}{ Sex, M vs F } & $1.26(0.78-2.03)$ & $1.49(1.00-2.21)$ & 0.22 & 0.05 & & \\
\hline & $1.72(1.09-2.74)$ & & & & & \\
\hline \multirow{2}{*}{$\begin{array}{l}\text { Duration of clinical history, > or } \\
<6 \text { mos }\end{array}$} & $0.76(0.48-1.21)$ & $2.20(1.47-3.31)$ & $<0.01$ & $<0.01$ & $1.00(0.52-1.97)$ & \multirow[t]{2}{*}{$<0.01$} \\
\hline & $20.11(7.84-51.55)$ & & & & $23.84(8.25-68.90)$ & \\
\hline \multirow[t]{2}{*}{ Seizures, generalized vs focal } & $96.10(25.60-855.51)$ & 300.58 (41.26-2189.83) & 0.43 & $<0.01$ & 354.13 (47.36-2647.89) & \multirow[t]{2}{*}{$<0.01$} \\
\hline & 314.63 (44.67-infinite) ${ }^{*}$ & & & & & \\
\hline \multirow[t]{2}{*}{ Side, rt vs It } & $0.71(0.44-1.16)$ & $1.43(0.71-1.25)$ & 0.03 & 0.10 & & \\
\hline & $1.25(0.79-1.97)$ & & & & & \\
\hline Location & & & $<0.01$ & $<0.01$ & & $<0.01$ \\
\hline \multirow[t]{2}{*}{ Parietal vs frontal } & $1.73(0.89-3.38)$ & $0.75(0.42-1.34)$ & & & $1.41(0.54-3.66)$ & \\
\hline & $0.26(0.11-0.63)$ & & & & $0.24(0.08-0.75)$ & \\
\hline \multirow[t]{2}{*}{ Insula vs frontal } & $2.78(1.53-5.05)$ & $1.42(0.86-2.36)$ & & & $1.79(0.84-3.82)$ & \\
\hline & $0.75(0.41-1.39)$ & & & & $0.37(0.17-0.83)$ & \\
\hline \multirow[t]{2}{*}{ Temporal vs frontal } & $1.19(0.60-2.38)$ & $0.92(0.54-1.57)$ & & & $0.61(0.24-1.53)$ & \\
\hline & $0.78(0.43-1.44)$ & & & & $0.57(0.22-1.48)$ & \\
\hline \multirow[t]{2}{*}{ Dominant side, no vs yes } & $0.74(0.46-1.19)$ & $0.96(0.65-1.41)$ & 0.06 & 0.83 & & \\
\hline & $1.21(0.77-1.90)$ & & & & & \\
\hline \multirow[t]{2}{*}{ Eloquent area, no vs yes } & $0.88(0.31-2.48)$ & $1.45(0.66-3.17)$ & 0.09 & 0.35 & & \\
\hline & $2.01(0.86-4.68)$ & & & & & \\
\hline \multirow[t]{2}{*}{ Corpus callosum, yes vs no } & $5.93(2.90-12.15)$ & $1.96(1.27-3.02)$ & $<0.01$ & $<0.01$ & $8.93(3.80-21.01)$ & \multirow[t]{2}{*}{$<0.01$} \\
\hline & $1.06(0.66-1.70)$ & & & & $1.73(0.82-4.64)$ & \\
\hline Volume & & & 0.87 & 0.85 & & \\
\hline \multirow[t]{2}{*}{ Cubic spline 1.1 vs 2.1} & $1.00(0.99-1.01)$ & $1.00(0.99-1.01)$ & & & & \\
\hline & $1.00(0.99-1.02)$ & & & & & \\
\hline \multirow[t]{2}{*}{ Cubic spline 1.2 vs 2.2} & $1.00(0.98-1.02)$ & $1.00(0.98-1.02)$ & & & & \\
\hline & $1.00(0.97-1.02)$ & & & & & \\
\hline \multirow[t]{2}{*}{ Border, well-defined vs irregular } & $0.47(0.27-0.82)$ & $0.52(0.34-0.81)$ & 0.54 & $<0.01$ & $0.46(0.24-0.89)$ & \multirow[t]{2}{*}{0.02} \\
\hline & $0.57(0.34-0.96)$ & & & & & \\
\hline \multirow{2}{*}{$\begin{array}{l}\text { Integrated diagnosis, oligoden- } \\
\text { droglioma vs astrocytoma }\end{array}$} & $1.38(0.87-2.19)$ & $1.72(1.17-2.53)$ & 0.09 & 0.01 & $1.83(1.02-3.30)$ & \multirow[t]{2}{*}{0.04} \\
\hline & $2.12(1.34-3.37)$ & & & & & \\
\hline \multirow[t]{2}{*}{ Grade, high vs low } & $0.96(0.57-1.63)$ & $0.56(0.35-0.89)$ & $<0.01$ & $<0.01$ & $1.01(0.50-2.06)$ & \multirow[t]{2}{*}{$<0.01$} \\
\hline & $0.26(0.13-0.52)$ & & & & $0.23(0.10-0.54)$ & \\
\hline \multirow[t]{2}{*}{ IDH1, wild-type vs mutation } & $0.26(0.09-0.76)$ & $0.47(0.24-0.95)$ & 0.08 & 0.03 & $1.14(0.37-3.53)$ & \multirow[t]{2}{*}{0.82} \\
\hline & $0.69(0.32-1.48)$ & & & & & \\
\hline \multirow{2}{*}{$\begin{array}{l}\text { Previous recurrence or biopsy, } \\
\text { yes vs no }\end{array}$} & $0.55(0.30-1.01)$ & $0.71(0.55-0.31)$ & 0.16 & $<0.01$ & $0.32(0.15-0.68)$ & $<0.01$ \\
\hline & $0.32(0.16-0.63)$ & & & & & \\
\hline
\end{tabular}

For each variable, the table reports: 1) the OR of the probability of partial/subtotal vs total resection, and the OR of the probability of supratotal vs total resection, and their $95 \% \mathrm{Cls} ; 2$ ) the OR under the constraint of equality; 3 ) the $p$ value for the hypothesis of equality; 4 ) the $p$ value for the inclusion of the variable into the regression model (using the proportional or the unconstrained model according to the result of the equality test); 5) the OR of the multivariable model; and 6 ) the $p$ value of the multivariable model.

*Penalized multinomial logistic regression.

surements of the degree of supratotal resection observed in deep (e.g., paralimbic) or posterior (e.g., posterior-temporal) locations, where it should be theoretically more crucial, to those obtained in frontal locations, where the influence of cavity collapse is less prominent. In deep locations, there was no evidence of a significant difference related to the degree of supratotal resection with respect to frontal locations (mean difference 7.82, $\mathrm{p}=0.31$ ), and this was independent from tumor volume. The same was observed for the affected hemisphere (mean difference $10.34, \mathrm{p}=0.14$ ).

\section{Impact on Functional Outcome}

The analysis of impact on functional outcome was per- 


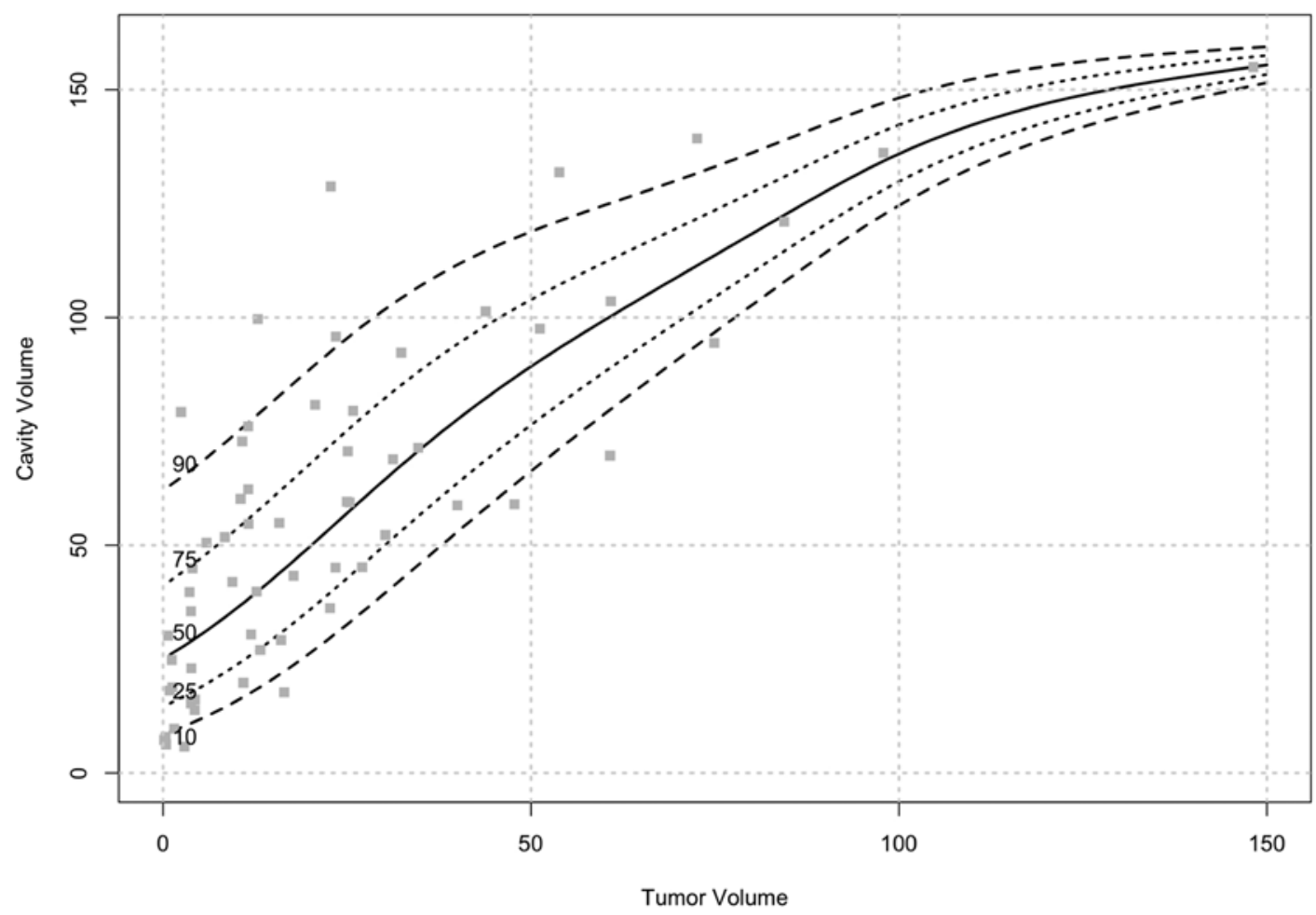

FIG. 3. Graph of the association between tumor volume $\left(\mathrm{cm}^{3}\right)$ and cavity volume $\left(\mathrm{cm}^{3}\right)$. A regression model with 3 spline bases was selected using AIC. The adjusted $\mathrm{R}^{2}$ value was 0.63 corrected for optimism. The plot shows the actual data (gray squares), the regression estimates of the median (solid line), quartiles (dotted lines), and 10th and 90th percentiles (dashed lines), according to generalized additive models for location, scale, and shape. The estimates show that cavity volume approaches tumor volume for large tumors, while for small tumors the cavity is larger than the tumor volume. Variability (distance between the quartiles or between 10th and 90th percentiles) is also larger for small tumors than for large tumors.

formed on a group of patients with total and supratotal resection for whom we had a complete set of neuropsychological profiles and QOL data before surgery, immediately after surgery, and at specific time points for follow-up. This pool included 100 patients, with comparable clinical and tumor parameters (Table 4).

\section{Impact on Neuropsychological Profile}

Neuropsychological tests were aggregated into five main cognitive domains: memory, language, praxis, attentive-executive functions, and fluid intelligence. The proportion of abnormal performance (equivalent score of 0 or 1) in the total and supratotal resection groups at three different time points (preoperative, postoperative, and 3 months postoperative) was compared. Memory, language, and fluid intelligence scores did not differ between groups, showing a dramatic decrease in mean scores immediately after surgery, followed by a substantial recovery after 3 months (Fig. 4A, B, and E). For praxis, a better performance immediately after surgery was observed in patients belonging to the total group in comparison to the supratotal group, although this difference reversed after 3 months (Fig. 4C). Patients belonging to the supratotal group experienced a better recovery of executive functions (Fig. 4D). Previous surgery or molecular profile did not affect any cognitive domain analyzed. Patient sex appeared to have a significant effect on the speed of language recovery, with female patients having a better score immediately after surgery in comparison to males, and a faster speed of recovery. At long-term follow-up (1 year), scores were also comparable (Fig. 5).

\section{Impact on QOL and Seizure Control}

QOL was evaluated in all patients, considering the global performance status and ability and time to return to work, with no significant difference existing between the two groups (Table 4). A more detailed analysis, by using the Hospital Anxiety and Depression Scale, conducted in 60 patients belonging to both groups, confirmed no difference.

Regarding the impact on seizure control, the two groups were comparable before surgery for occurrence of seizures $\left(80 \%\right.$ vs $\left.87.5 \%, \chi^{2}[1]=0.33, p=0.56\right)$ and administration of AEDs. After surgery, we observed a significant improvement of epilepsy control in the supratotal group $\left(91.7 \%\right.$ vs $77.5 \%, \chi^{2}[1]=4.089, \mathrm{p}<0.05$; Table 4$)$.

\section{Discussion}

This work addresses separate issues: 1) the feasibility in clinical practice of supratotal resection in LGG; 2) the factors associated with its achievement; 3 ) the degree of 
TABLE 4. Clinical and imaging features of patients belonging to the prospective group used for the detailed neuropsychological analysis of the impact of EOR on patient performance

\begin{tabular}{|c|c|c|c|}
\hline Variable & Total & Supratotal & Statistical Analysis \\
\hline No. of patients & 40 & 60 & \\
\hline Mean age, yrs & 36.86 & 38.2 & $p=0.551$ \\
\hline \multicolumn{4}{|l|}{ Sex, $n(\%)$} \\
\hline Male & $24(60)$ & $30(50)$ & \\
\hline Female & $16(40)$ & $30(50)$ & \\
\hline Tumor location, $\mathrm{n}(\%)$ & & & $\chi^{2}(1)=0, p=0.1$ \\
\hline Dominant side & $24(60)$ & $34(57)$ & \\
\hline Nondominant side & $16(40)$ & $26(43)$ & \\
\hline Lobe, $n(\%)$ & & & $\chi^{2}(3)=0, p=0.107$ \\
\hline Frontal & $16(40)$ & $28(46.7)$ & \\
\hline Parietal & $8(20)$ & $4(6.7)$ & \\
\hline Temporal & $1(2.5)$ & 7 (11.7) & \\
\hline Paralimbic & $15(37.5)$ & $21(35)$ & \\
\hline \multicolumn{4}{|l|}{ Preop tumor volume, $\mathrm{cm}^{3}$} \\
\hline Mean & 29.78 & 24.43 & $p=0.32$ \\
\hline Min & 1.08 & 1.7 & \\
\hline Max & 100.72 & 148.17 & \\
\hline EOR & $100 \%$ & $>100 \%(665 \%)$ & \\
\hline Recurrent or previously biopsied tumors, $n(\%)$ & $6(15)$ & $9(15)$ & $\chi^{2}(1)=0.62, p=0.43$ \\
\hline Epilepsy control presurgery, n (\%) & $35(87.5)$ & $48(80)$ & $\chi^{2}(1)=0.33, p=0.56$ \\
\hline No. of AEDs taken preop, $\mathrm{n}(\%)$ & & & $\chi^{2}(1)=0.98, p=0.32$ \\
\hline 1 & $25(62.5)$ & $31(51.7)$ & \\
\hline$\geq 2$ & $8(20)$ & $10(16.7)$ & \\
\hline Neurological deficit/symptoms, $n(\%)^{*}$ & $5(12.5)$ & $8(13.3)$ & $\chi^{2}(1)=0.0096, p=0.921$ \\
\hline Motor & 1 & 1 & \\
\hline Sensory & 2 & 2 & \\
\hline Apraxia & 1 & 0 & \\
\hline Language & 0 & 1 & \\
\hline Headache & 1 & 4 & \\
\hline Vertigo & 0 & 2 & \\
\hline Global performance status at 3-mo follow-up $\dagger$ & 90 & 90 & \\
\hline Ability to work at 3 mos, $n(\%)$ & $39(97.5)$ & $58(96.7)$ & \\
\hline Days to return to work & 46 & 48 & \\
\hline Epilepsy control 3 mos after surgery, $n(\%)$ & $31(77.5)$ & $55(91.7)$ & $\chi^{2}(1)=4.089, p<0.05$ \\
\hline No. of AEDs in patients with 3-mo postop seizure control, $n(\%)$ & & & $\chi^{2}(1)=6.418, p<0.05$ \\
\hline 1 & $14(48.3)$ & $41(78.8)$ & \\
\hline$\geq 2$ & $15(51.7)$ & $11(21.2)$ & \\
\hline
\end{tabular}

* Recorded at admission.

† Score range 10-100.

supratotal resection achieved; and 4) the general safety and functional impact of the procedure.

The first issue involves the main criticism attributed to supratotal resection, that it is generally considered to be feasible in only a few cases, in highly favorable and not critically eloquent locations. We reviewed all cases harboring a presumptive radiological diagnosis of LGG consecutively admitted to our service in a 5-year period, and candidates for resective surgery targeted to reach functional boundaries, without any a priori selection. In this large series, a supratotal resection was reached in $32.3 \%$ of cases, and a total resection in $40.8 \%$. These data indicate that a supratotal resection can be achieved in a considerable number of patients in the clinical routine when a functional approach is used.

The second issue concerns the identification of putative factors associated with the achievement of a supratotal resection. We investigated parameters associated with patient or clinical history, others deducible from preoperative conventional MRI, others available intraoperatively, and others linked to the tumor. There was no evidence of an association with preoperative clinical features or imaging factors. The effect of tumor volume, proximity of eloquent sites, or tumor border appearance were not as 


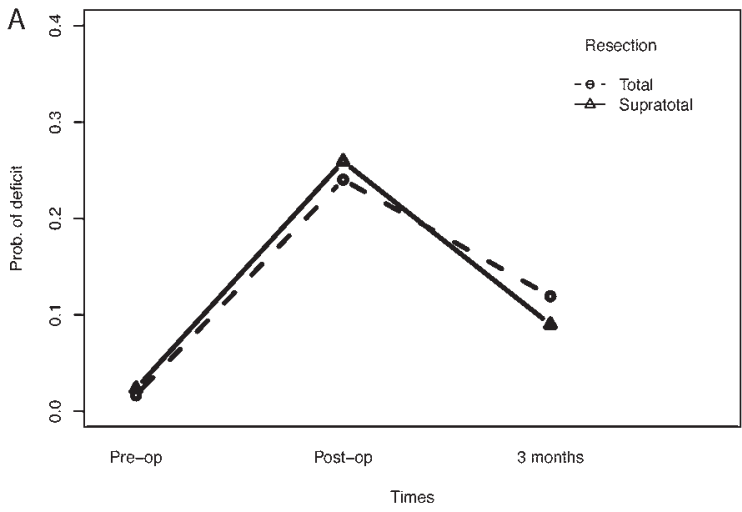

Language

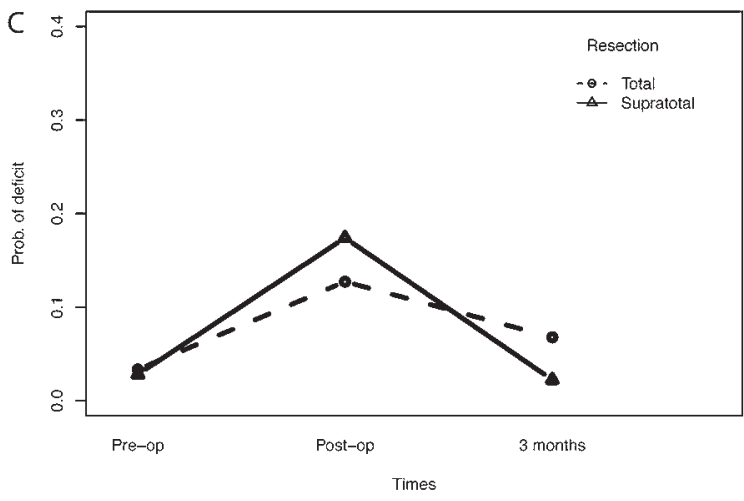

Apraxia

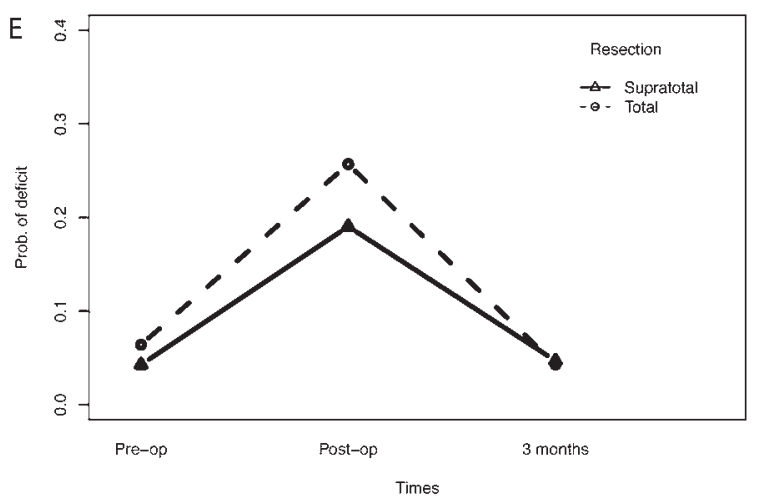

Fluid intelligence

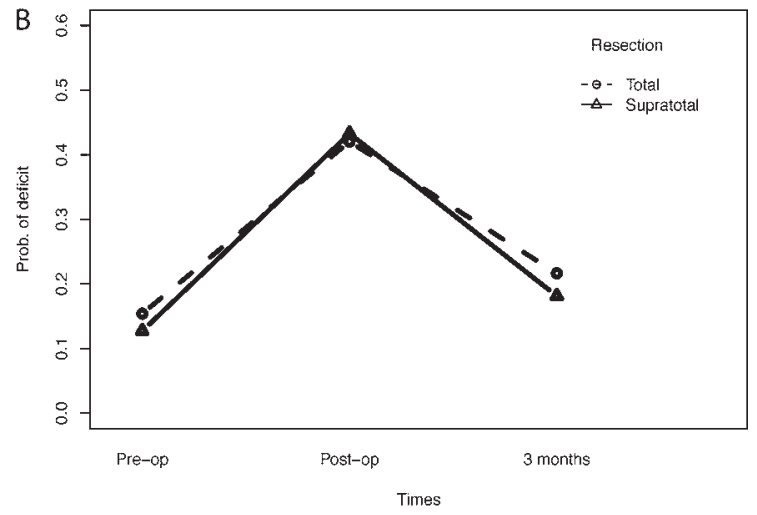

Memory

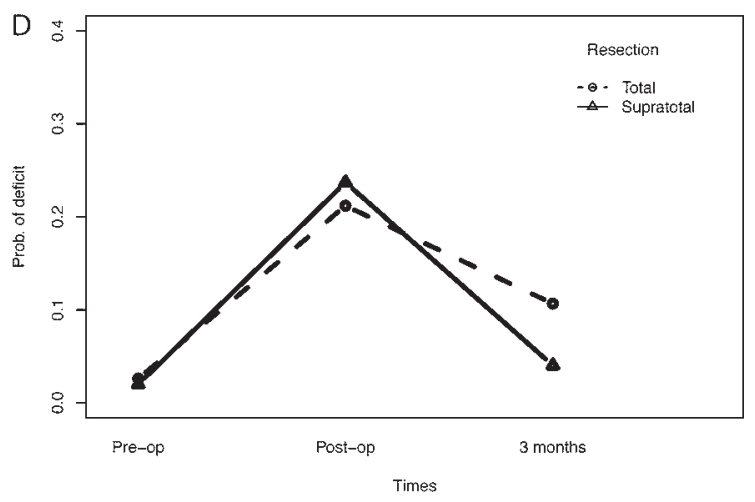

Attentive and Executive functions

\begin{tabular}{|l|l|}
\hline Language & $\begin{array}{l}\text { Time effect } z=-4.72 p<0.001 \\
\text { Resection } z=0.31 p=0.758 \\
\text { Linear interaction } z=-1.51 p=0.132 \\
\text { Quadratic interaction } z=0.01 p=0.992\end{array}$ \\
\hline Memory & $\begin{array}{l}\text { Time effect } z=-3.69 p<0.001 \\
\text { Resection } z=-0.58 p=0.56 \\
\text { Linear interaction } z=-0.23 p=0.821 \\
\text { Quadratic interaction } z=-1.67 p=0.095\end{array}$ \\
\hline Apraxia & $\begin{array}{l}\text { Time effect } z=0.80 p=0.422 \\
\text { Resection } z=-1.97 p=0.049 \\
\text { Linear interaction } z=-0.81 p=0.42 \\
\text { Executive Functions } \\
\text { Quadratic interaction } z=-1.73 p=0.083\end{array}$ \\
& $\begin{array}{l}\text { Time effect } z=3.13 p=0.002 \\
\text { Resection } z=-0.86 p=0.391 \\
\text { Linear interaction } z=-0.23 p=0.821 \\
\text { Quadratic interaction } z=-5.00 p<0.001\end{array}$ \\
\hline Fluid intelligence & $\begin{array}{l}\text { Time effect } z=-3.69 p<0.001 \\
\text { Resection } z=-0.58 p=0.56 \\
\text { Linear interaction } z=-0.23 p=0.821 \\
\text { Quadratic interaction } z=-1.67 p=0.095\end{array}$ \\
\hline
\end{tabular}

FIG. 4. Neuropsychological profile in patients who underwent total or supratotal resection. Probability (Prob.) of deficits according to the time point for each functional domain tested: language $(A)$, memory $(B)$, apraxia (C), attentive-executive functions (D), and fluid intelligence $(E)$. The proportion of abnormal deficits is on the y-axis (abnormal performance = equivalent score of 0 or 1). Time points were preoperative (Pre-op), at 5 days postoperative (Post-op), and at 3 months postoperative (x-axis). Results are reported for patients submitted to total (dashed line) or supratotal (solid line) resection. Detailed statistics for each functional domain are also given (F). Time effect and resection indicate the effect exerted by time and resection on each functional domain. The linear and quadratic interactions are also reported.

clinically relevant. The achievement of supratotal resection was instead associated with a clinical history longer than 6 months, a histological diagnosis of a low-grade tumor, and an oligodendroglial feature. Regarding the effect of location, the likelihood of reaching a total or suprato- tal resection was higher in frontal and temporal tumors, but without evidence of a significant difference between total or supratotal resection groups. Considered together, these data suggest that a supratotal resection is achieved in tumors in which a long clinical history and slow tumor 

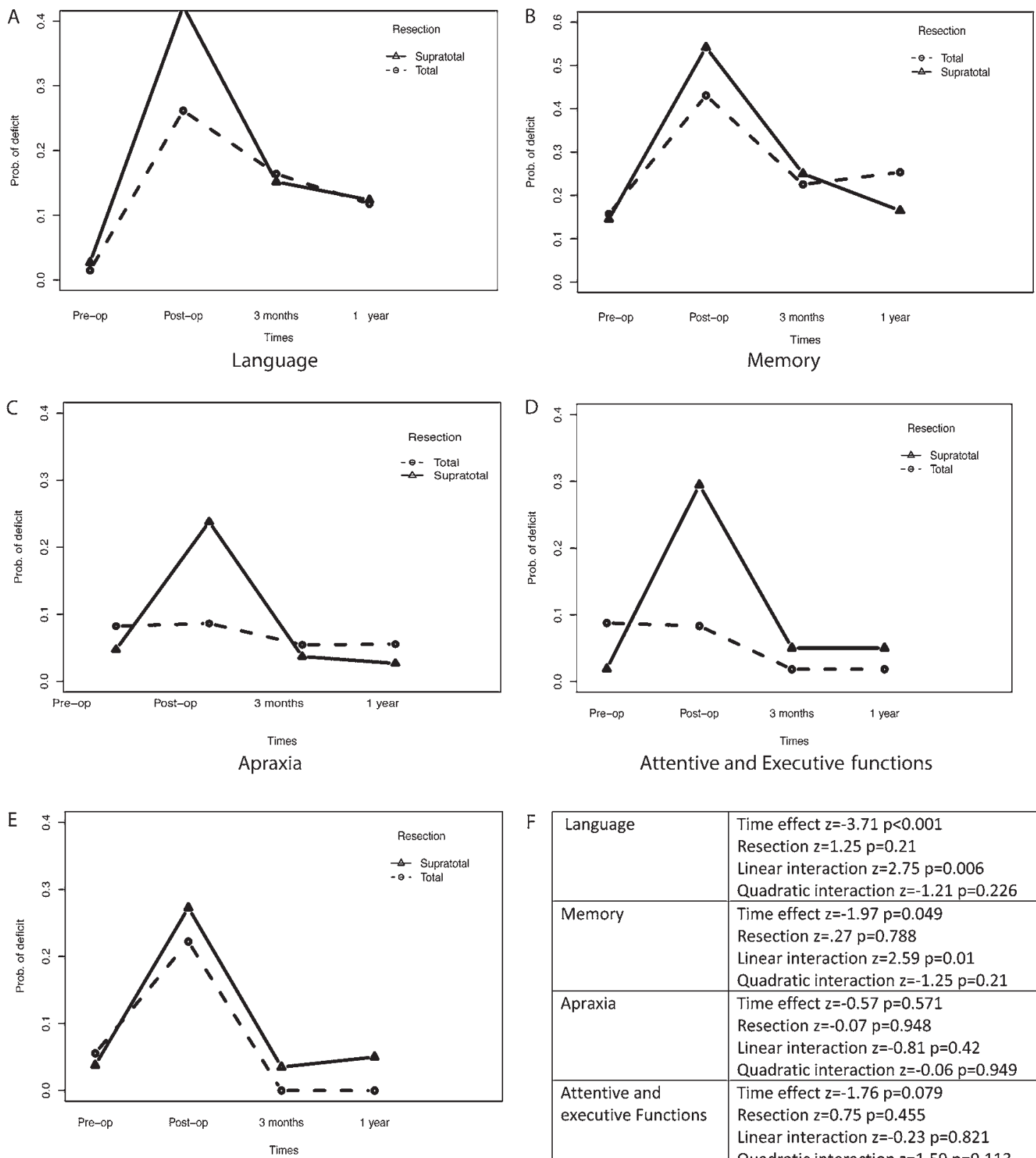

\begin{tabular}{|l|l|}
\hline Language & $\begin{array}{l}\text { Time effect } z=-3.71 p<0.001 \\
\text { Resection } z=1.25 p=0.21 \\
\text { Linear interaction } z=2.75 p=0.006 \\
\text { Quadratic interaction } z=-1.21 p=0.226\end{array}$ \\
\hline Memory & $\begin{array}{l}\text { Time effect } z=-1.97 p=0.049 \\
\text { Resection } z=.27 p=0.788 \\
\text { Linear interaction } z=2.59 p=0.01 \\
\text { Quadratic interaction } z=-1.25 p=0.21\end{array}$ \\
\hline Apraxia & $\begin{array}{l}\text { Time effect } z=-0.57 p=0.571 \\
\text { Resection } z=-0.07 p=0.948 \\
\text { Linear interaction } z=-0.81 p=0.42 \\
\text { Quadratic interaction } z=-0.06 p=0.949\end{array}$ \\
\hline Attentive and & $\begin{array}{l}\text { Time effect } z=-1.76 p=0.079 \\
\text { executive Functions } \\
\end{array}$ \\
& $\begin{array}{l}\text { Linear interaction } z=-0.23 p=0.821 \\
\text { Quadratic interaction } z=1.59 p=0.113\end{array}$ \\
\hline Fluid intelligence & $\begin{array}{l}\text { Time effect } z=-0.0002 p=1 \\
\text { Resection } z=0.0008, p=0.999 \\
\text { Linear interaction } z=0.0007 p=0.999 \\
\text { Quadratic interaction } z=-0.0006 p=1\end{array}$ \\
\hline
\end{tabular}

FIG. 5. Neuropsychological profile in patients who underwent total or supratotal resection. Probability of deficits according to time for each functional domain in the group of patients with longer neuropsychological follow-up: language (A), memory (B), apraxia (C), attentive-executive functions (D), and fluid intelligence (E). The proportion of abnormal deficits is on the y-axis (abnormal performance $=$ equivalent score of 0 or 1 ). Time points were preoperative (Pre-op), at 5 days postoperative (Post-op), and at 3 and 12 months postoperative (x-axis). Results are reported for patients submitted to total (dashed line) or supratotal (solid line) resection. Detailed statistics for each functional domain are also given $(\mathbf{F})$. Time effect and resection indicate the effect exerted by time and resection on each functional domain. The linear and quadratic interactions are also reported.

growth induce a high degree of functional reorganization of subcortical networks present in the tumor area. ${ }^{11,12}$ The existence of such a high degree of functional reshaping enables identification of all functional boundaries beyond the MRI-visible tumor border at surgery, a further param- eter that strongly characterized supratotal resection cases. In patients in whom a supratotal resection was achievable, the existence of such a high functional network rebalance is confirmed by the fact that this surgery, in comparison to other resections, did not require a longer surgical or 
mapping time, and was not associated with higher patient fatigue, but was dependent on the location of functional tracts with respect to MRI-visible tumor margins.

The third issue concerns the degree of supratotal resection. When a supratotal resection is feasible, a consistent amount (median 320\%) of tumor surrounding parenchyma could be removed, independent of location or affected hemisphere. Although preoperative tumor volume did not influence the chance of achieving a total versus supratotal resection, the amount of tissue removed was associated with tumor volume, being progressively less when the tumor volume increased. This is not surprising, because the larger the tumor volume, the larger the number of functional networks involved that may possibly be found at tumor margins.

The fourth issue concerns the safety of the procedure. The analysis did not show evidence of a significant difference among resection groups, and globally all resections were safe. Rates of new immediate deficits were quite high and independent from the EOR achieved, as expected when functional boundaries are always reached at the subcortical level. Most deficits recovered in a few weeks. The safety of the procedure is also supported by the analysis of surgically related complications. The rate of deficits or complications registered in partial or subtotal resection was higher, suggesting that persistence of tumors in the surgical cavity may expose the patient to immediate or delayed complications.

The impact on functional outcome was evaluated by a detailed neuropsychological and QOL evaluation. ${ }^{15}$ Considering the complexity of the investigation, the analysis was performed in a patient subgroup commensurate for clinical and imaging variables. Various functional domains were analyzed, using a battery specifically designed for patients with LGG. ${ }^{20}$ Overall, in all cognitive tests administered, patients with total and supratotal resection showed a very similar pattern in score means: starting from comparable scores in the preoperative period, both displayed an important decline during the first week, followed by a substantial recovery at 1 month..$^{5,7,10,12}$ At 3 months, the majority of patients were able to restart work and a normal life. This positive trend of recovery was further confirmed by the scores obtained at the 1 -year evaluation, in which patients reported equal or better scores than those observed at 1-3 months after surgery. The speed of recovery regarding language was quicker in females, possibly linked to the more bilateral organization of language tracts in their brains..$^{13}$ Globally, this indicates that both approaches were maintaining a long-term and high level of cognitive performance. A difference was instead observed for the rate of seizure control. A consistent decrease in the number and frequency of seizures was found in the supratotal group. In this context, the number of AEDs required to obtain seizure control was also lower, indicating that removal of brain parenchyma surrounding the tumor (where the irritative zone may be located) affords better epileptic long-term control, particularly in cases of long-standing and intractable epilepsy. ${ }^{25}$

The current study has limitations. The achievement of distant functional boundaries extending resection raises the need for expanding the current battery of mapping tests, adding more sophisticated tests for detecting complex cognitive functions. Similarly, the neuropsychological battery should be added with a more complete evaluation for exploring the global QOL and psycho-oncological profile.

The fact that the surgical cavity could collapse after resection, particularly in some locations such as those posterior, might underestimate the number of cases in which a supratotal resection was achieved, and globally decrease its degree. The calculation of the degree of supratotal resection was performed by applying the method most often used for performing such a calculation. ${ }^{25}$ In this case, the degree of supratotal resection did not differ between anterior and posterior locations, and was not dependent upon tumor volume or affected hemisphere. Additional methods may be applied to further explore this point.

\section{Conclusions}

Globally, this is the first report describing the feasibility and safety of supratotal resection of LGGs in routine practice. The ability to reach a supratotal resection apparently depends on the level of functional reorganization reached by the individual patient's brain and is predicted by a long clinical history.

\section{Acknowledgments}

The work was supported by grants to L.B. by Fondazione Berlucchi and AIRC (grant no. 18482).

\section{References}

1. Aboian MS, Solomon DA, Felton E, Mabray MC, VillanuevaMeyer JE, Mueller S, et al: Imaging characteristics of pediatric diffuse midline gliomas with histone H3 K27M mutation. AJNR Am J Neuroradiol 38:795-800, 2017

2. Aghi MK, Nahed BV, Sloan AE, Ryken TC, Kalkanis SN, Olson JJ: The role of surgery in the management of patients with diffuse low grade glioma: A systematic review and evidence-based clinical practice guideline. J Neurooncol 125:503-530, 2015

3. Ananth CV, Kleinbaum DG: Regression models for ordinal responses: a review of methods and applications. Int J Epidemiol 26:1323-1333, 1997

4. Bayen RH, Davidson DJ, Bates DM: Mixed-effects modeling with crossed random effects for subjects and items. J Mem Lang 10:542-545, 2008

5. Bello L, Fava E, Carrabba G, Papagno C, Gaini SM: Present day's standards in microsurgery of low-grade gliomas. Adv Tech Stand Neurosurg 35:113-157, 2010

6. Bello L, Gambini A, Castellano A, Carrabba G, Acerbi F, Fava E, et al: Motor and language DTI fiber tracking combined with intraoperative subcortical mapping for surgical removal of gliomas. Neuroimage 39:369-382, 2008

7. Bello L, Riva M, Fava E, Ferpozzi V, Castellano A, Raneri F, et al: Tailoring neurophysiological strategies with clinical context enhances resection and safety and expands indications in gliomas involving motor pathways. Neuro Oncol 16:1110-1128, 2014

8. Buckner J, Giannini C, Eckel-Passow J, Lachance D, Parney I, Laack N, et al: Management of diffuse low-grade gliomas in adults-use of molecular diagnostics. Nat Rev Neurol 13:340-351, 2017

9. Cascino GD: Epilepsy and brain tumors: implications for treatment. Epilepsia 31 (Suppl 3):S37-S44, 1990 
10. De Witt Hamer PC, Robles SG, Zwinderman AH, Duffau H, Berger MS: Impact of intraoperative stimulation brain mapping on glioma surgery outcome: a meta-analysis. J Clin Oncol 30:2559-2565, 2012

11. Duffau H: Long-term outcomes after supratotal resection of diffuse low-grade gliomas: a consecutive series with 11-year follow-up. Acta Neurochir (Wien) 158:51-58, 2016

12. Duffau H: A new philosophy in surgery for diffuse low-grade glioma (DLGG): oncological and functional outcomes. Neurochirurgie 59:2-8, 2013

13. Forkel SJ, Thiebaut de Schotten M, Dell'Acqua F, Kalra L, Murphy DGM, Williams SCR, et al: Anatomical predictors of aphasia recovery: a tractography study of bilateral perisylvian language networks. Brain 137:2027-2039, 2014

14. Hosmer DW, Lemeshow S: Applied Logistic Regression, ed 2. New York: Wiley, 2000

15. Klein M: Neurocognitive functioning in adult WHO grade II gliomas: impact of old and new treatment modalities. Neuro Oncol 14 (Suppl 4):iv17-iv24, 2012

16. Morrow AM, Morgan RM, Villano JL: Seizure control as a new metric in assessing efficacy of tumor treatment in lowgrade glioma trials-impact of psychogenic non-epileptic seizures. Neuro Oncol 19:1010-1011, 2017

17. Nabavi A, Black PM, Gering DT, Westin CF, Mehta V, Pergolizzi RS Jr, et al: Serial intraoperative magnetic resonance imaging of brain shift. Neurosurgery 48:787-798, 2001

18. Nayak L, DeAngelis LM, Brandes AA, Peereboom DM, Galanis E, Lin NU, et al: The Neurologic Assessment in Neuro-Oncology (NANO) scale: a tool to assess neurologic function for integration into the Response Assessment in Neuro-Oncology (RANO) criteria. Neuro Oncol 19:625635, 2017

19. Pallud J, Varlet P, Devaux B, Geha S, Badoual M, Deroulers $\mathrm{C}$, et al: Diffuse low-grade oligodendrogliomas extend beyond MRI-defined abnormalities. Neurology 74:1724-1731, 2010

20. Papagno C, Casarotti A, Comi A, Gallucci M, Riva M, Bello L: Measuring clinical outcomes in neuro-oncology. A battery to evaluate low-grade gliomas (LGG). J Neurooncol 108:269-275, 2012

21. Rigby RA, Stasinopoulos DM, Lane PW: Generalized additive models for location, scale and shape. J R Stat Soc Ser C Appl Stat 16:513, 2005

22. Riva M, Hennersperger C, Milletari F, Katouzian A, Pessina F, Gutierrez-Becker B, et al: 3D intra-operative ultrasound and MR image guidance: pursuing an ultrasound-based management of brainshift to enhance neuronavigation. Int J CARS 12:1711-1725, 2017
23. Schucht P, Knittel S, Slotboom J, Seidel K, Murek M, Jilch A, et al: 5-ALA complete resections go beyond MR contrast enhancement: shift corrected volumetric analysis of the extent of resection in surgery for glioblastoma. Acta Neurochir (Wien) 156:305-312, 2014

24. Steyerberg EW: Book Reviews. FRANK E. HARRELL, Jr., Regression Modeling Strategies: With Applications, to Linear Models, Logistic and Ordinal Regression, and Survival Analysis, 2nd ed. Heidelberg: Springer. Biometrics 72:10061007, 2016

25. van den Bent MJ, Wefel JS, Schiff D, Taphoorn MJB, Jaeckle $\mathrm{K}$, Junck L, et al: Response assessment in neuro-oncology (a report of the RANO group): assessment of outcome in trials of diffuse low-grade gliomas. Lancet Oncol 12:583-593, 2011

26. Yordanova YN, Moritz-Gasser S, Duffau H: Awake surgery for WHO Grade II gliomas within "noneloquent" areas in the left dominant hemisphere: toward a "supratotal" resection. Clinical article. J Neurosurg 115:232-239, 2011

\section{Disclosures}

The authors report no conflict of interest concerning the materials or methods used in this study or the findings specified in this paper.

\section{Author Contributions}

Conception and design: Rossi. Acquisition of data: Rossi, Gay, Conti Nibali, Leonetti, Puglisi, Sciortino, Navarria, Franzese, Simonelli. Analysis and interpretation of data: Gay, Conti Nibali, Bello. Drafting the article: Rossi, Gay, Bello. Critically revising the article: Rossi, Leonetti, Puglisi, Riva, Pessina, Navarria, Rudà, Bello. Reviewed submitted version of manuscript: Rossi, Howells, Bello. Statistical analysis: Ambrogi, Gay, Gallucci. Study supervision: Rudà, Bello.

\section{Supplemental Information}

\section{Previous Presentations}

The work was partially presented as an oral presentation at the 2018 European Association of Neurosurgical Societies meeting.

\section{Correspondence}

Marco Rossi: Humanitas Research Hospital, Milan, Italy. rossi. marco@gmail.com. 